\title{
A Comparative Study of Efficacy and Tolerability of Solifenacin and Mirabegron, for the Treatment of Overactive Bladder - A Randomized Prospective Control Study
}

\author{
Senthil Kumar Thiagarajann', Vikrant Mahajan², Jambunathan Saravanann ${ }^{3}$, Harsha Kurabalakota ${ }^{4}$, \\ RM Meyyappan ${ }^{5}$, T Srinavasan ${ }^{6}$, Madhu Gowda ${ }^{7}$, Gokul Nachiketh ${ }^{8}$ \\ 1, 2, 3, 4, 5, 6, 7, 8 Department of Urology, SRM Medical College Hospital and Research Centre, SRM Institute of \\ Science and Technology, Potheri, Kattankulathur, Chengalpattu District, Tamil Nadu, India.
}

\section{ABSTRACT}

\section{BACKGROUND}

Overactive bladder $(\mathrm{OAB})$ is a chronic, age-related disorder seen in $11 \%$ of patients. Symptoms consist of urinary urgency, with or without urinary incontinence, usually with frequency or nocturia. The objective of the present study was to compare the efficacy and side effects of mirabegron and solifenacin as primary therapies in patients with overactive bladder.

\section{METHODS}

This was a prospective interventional study. 100 patients aged between 18 years and 50 years with overactive bladder were included and were assigned into two treatment groups of solifenacin $5 \mathrm{mg}$ or mirabegron $50 \mathrm{mg}$. They were asked to record the number of micturitions in a day, urgency episodes, incontinence episodes and volume of each micturition. All patients went through a basic workup with blood sugar to rule out diabetes, USG KUB to rule out bladder stones, and urine culture and sensitivity to rule out urinary tract infection (UTI).

\section{RESULTS}

100 patients with $\mathrm{OAB}$ were selected for the study and divided into equal groups, 50 receiving $5 \mathrm{mg}$ solifenacin and 50 receiving $50 \mathrm{mg}$ mirabegron. Both groups increased the mean micturition volume but mirabegron was more effective in increasing the mean micturition in patients with OAB. Both drugs were well tolerated. There was a significant increase in mean micturition volume in mirabegron $50 \mathrm{mg}$ group (by $20.7+/-2.2 \mathrm{~mL}$ ), $\mathrm{P}<0.001$ whereas in solifenacin group micturition volume was increased to $22.2+/-0.97 \mathrm{ml}$ ). The most common side-effect in the mirabegron group was hypertension and the most common side effect in the solifenacin group was dry mouth.

\section{CONCLUSIONS}

Both mirabegron and solifenacin were effective in controlling the frequency of micturition, decreasing urgency and incontinence episodes and increasing the mean volume of micturition. Mirabegron was more effective than solifenacin in controlling urgency and incontinence episodes and increasing the mean volume of micturition.

\section{KEY WORDS}

Overactive Bladder (OAB), Micturition, Mirabegron, Solifenacin.
Corresponding Author: Dr. Vikrant Mahajan, Room No. 416, PG Men's Hostel, SRM Medical College, Potheri - 603203, Kanchipuram, Tamil Nadu, India.

E-mail: vikrantmahajan2800@gmail.com

DOI: $10.14260 / \mathrm{jemds} / 2021 / 741$

How to Cite This Article:

Thiagarajan SK, Mahajan V, Saravanan J, et al. A comparative study of efficacy and tolerability of solifenacin and mirabegron, for the treatment of overactive bladder - $a$ randomized prospective control study. J Evolution Med Dent Sci 2021;10(42):36543658, DOI: 10.14260/jemds/2021/741

Submission 10-03-2021,

Peer Review 20-06-2021

Acceptance 26-06-2021,

Published 18-10-2021.

Copyright (C) 2021 Senthil Kumar Thiagarajan et al. This is an open access article distributed under Creative Commons Attribution License [Attribution 4.0 International (CC BY 4.0)] 


\section{BACKGROUND}

Storage symptoms are experienced during the storage phase of the bladder and include any daytime frequency and nocturia. ${ }^{1}$ Overactive bladder is a symptomatic condition defined as urgency, with or without urinary incontinence usually with frequency and nocturia. ${ }^{2}$ Overactive bladder $(\mathrm{OAB})$ is a chronic, age-related disorder seen in $11.8 \%$ of men and $28.7 \%$ of women. 3,4 The prevalence of symptoms of the overactive bladder occurs in women in their late 40's and men in their $5^{\text {th }}$ decade. The pathophysiology appears to be multifactorial with multiple factors like sensory activity, motor control and reflexes of the lower urinary tract. Two hypotheses have been postulated namely the urothelium based hypothesis and the myogenic hypothesis. In the urothelium based hypothesis, the various mechanical, chemical and inflammatory stimuli alter the sensitivity of cell membrane receptors and cause the release of chemical mediators that trigger bladder contractility. In the myogenic hypothesis there are contractions within the smooth muscle layer of the bladder and propagation to the whole bladder wall. Symptoms consist of urinary urgency, with or without urinary incontinence, usually with frequency or nocturia. Urinary incontinence is present in around $30 \%$ of cases and is most bothersome among all symptoms. These patients are evaluated by history and clinical examination to find out any medications or food habits responsible for overactive bladder. 5 They are categorized based on the bladder sensation scale used in the International Consultation On Incontinence Questionnaire bladder diary and patient perception of the intensity of urgency scale. ${ }^{6}$ Score ranges from 1 to 5 where.

1. No urgency

2. Mild urgency

3. Moderate urgency

4. Severe urgency and

5. Urgency incontinence.

After the categorization of patients based on scoring systems, they undergo urinalysis, blood sugar, urea creatinine, urine culture, lower abdomen ultrasound, uroflowmetry and if needed urodynamics in select cases having a high score in urgency scales. An array of management options from behavioural therapy, medical management and surgical management are available. Most patients show good improvement of symptoms on behavioural therapy, dietary modifications and pelvic floor strengthening exercises. ${ }^{7}$ Refractory cases that do not respond to antimuscarinic agents has been the mainstay of treatment. Drugs commonly used are antimuscarinics like oxybutynin, solifenacin, flavoxate, tolterodine, hyoscyamine, and darifenacin. As per EAU guidelines for patients with moderate to severe lower urinary tract symptoms, mainly overactive bladder, antimuscarinic agents are strongly recommended and they seem to improve urgency, urinary incontinence and daytime frequency and the level of recommendation in patients with significant post-void residual urine is weak. Mirabegron has similar efficacy but patients remained longer on treatment when compared to antimuscarinics. ${ }^{8,9} \mathrm{OAB}$ patients may have a suboptimal response or encounter side-effects such as dry mouth, constipation, and blurred vision resulting from muscarinic receptor blockade and urinary retention when used in men with prostatic enlargement. This might affect the persistence of therapy. Therefore, there is a need for a new treatment option for OAB that is effective and well-tolerated, with a different mechanism of action. Mirabegron belongs to a new class of agents developed for the $\mathrm{OAB}$ treatment. It's a selective $\beta 3$ - adrenergic receptor agonist. It causes relaxation of the detrusor muscle to increase bladder capacity during the storage phase. It doesn't impair detrusor contractions during the voiding phase of the micturition cycle. In this study, we compared the efficacy of solifenacin with mirabegron in OAB.

\section{METHODS}

This study was conducted at SRM University from August 2018 to September 2019 after obtaining ethical committee approval. This was a prospective non-randomised singleblinded interventional study. Based on previous studies our study population included 100 patients between 18 years and 50 years. The patients were alternately assigned into two treatment groups of solifenacin $5 \mathrm{mg}$ (Group 1, 50 patients) and mirabegron $50 \mathrm{mg}$ (Group 2, 50 patients). They were asked to record the number of micturitions in a day, urgency episodes, incontinence episodes and volume of each micturition. Patients were asked to void each time in a bowl or beaker to measure the volume. All patients went through a basic workup with FBS to rule out diabetes, USG KUB to rule out bladder stones, and urine culture and sensitivity to rule out UTI. The sample size was calculated using the following formula -

$\mathrm{n}=\frac{Z 2 \times S 2}{d 2}$

$\mathrm{Z}=$ Standardized normal deviation (1.96)

$\mathrm{S}=$ Standard deviation collected from previous published international studies (2.79) $\mathrm{d}=$ clinically expected variable $(0.8)^{10}$

\section{Inclusion Criteria}

Male and female patients of 18 years or older with overactive bladder symptoms like frequency, urgency, nocturia, urge incontinence for a month or more were enrolled in the study. At baseline, patients must have experienced an average of 8 or more micturitions per 24 hours and 3 or more urgency episodes with or without incontinence.

\section{Exclusion Criteria}

Patients with

1. Stress urinary incontinence which is a prominent symptom that is seen at the screening

2. An indwelling catheter

3. Symptomatic urinary tract infection

4. Chronic inflammation

5. Benign prostatic hyperplasia (BPH)

6. Diabetic cystopathy

7. Previous history of genitourinary tuberculosis (GUTB)

8. Bladder stones

9. Previous pelvic radiation therapy

10. Previous or current malignant disease of the pelvic organs. 
at week 4 and to $1.06+/-1.32$ at week 8 and to $0.68+/$ 0.98 per day at week 12 in the mirabegron group. This shows a decreasing trend of urgency episodes after initiation of therapy. The decrease in the urgency episodes from baseline to week 12 was significant as evident by the P-value of < 0.001 . This shows that mirabegron is effective in controlling the urgency episodes in a patient with OAB. There was a significant reduction in urgency episodes in mirabegron 50 mg (by $0.19+/-0.03$ ) compared to the placebo group (by $0.08+/$ - 0.03). Solifenacin group: Urgency episodes decreased from an average of $3.92+/-2.59$ per day at baseline to $2.64+/-2.07$ at week 4 and to $1.88+/-1.48$ at week 8 and to $1.7+/-1.53$ per day at week 12 . This shows a decreasing trend of urgency episodes after initiation of therapy. The decrease in the urgency episodes from baseline to week 12 was significant as evident by the P-value of < 0.001 . This shows that solifenacin is effective in controlling the urgency episodes in a patient with OAB. This is in accordance with the study conducted by Luo and colleagues which showed a significant decrease in the urgency episodes with solifenacin in patients with OAB. Mirabegron Vs Solifenacin: Though urgency episodes decreased in both the groups, the decrease in the mirabegron group was significantly more compared to the solifenacin group at week $4(\mathrm{P}<0.0001)$, week $8(\mathrm{P}=0.0278)$ and week $12(\mathrm{P}=$ $0.0114)$. So based on our present study, mirabegron is more effective in decreasing the urgency episodes in patients with OAB.

\section{Incontinence \\ Mirabegron Group}

Incontinence episodes decreased from an average of $1.40+/$ - 1.39 per day at baseline to $0.58+/-0.76$ at week 4 and to $0.42+/-0.64$ at week 8 and to $0.16+/-0.37$ per day at week 12 in the mirabegron group. This shows a decreasing trend of incontinence episodes after initiation of therapy. The decrease in the incontinence episodes from baseline to week 12 was significant as evident by the P-value of $<0.001$. This shows that mirabegron is effective in controlling the incontinence episodes in a patient with OAB. Solifenacin group: Incontinence episodes decreased from an average of $1.48+/-1.45$ per day at baseline to $0.92+/-1.05$ at week 4 and to $0.84+/-1.08$ at week 8 and to $0.68+/-0.87$ per day at week 12 in the solifenacin group. This shows a decreasing trend of incontinence episodes after initiation of therapy. The decrease in the incontinence episodes from baseline to week 12 was significant as evident by the P-value of $<0.001$. Mirabegron Vs Solifenacin: Though incontinence episodes decreased in both the groups, the decrease in the mirabegron group was significantly more compared to the solifenacin group at week $4(\mathrm{P}<0.0155)$, week $8(\mathrm{P}<0.0045)$ and week $12(\mathrm{P}<0.0088)$. So based on our present study, mirabegron is more effective in decreasing the incontinence episodes in patients with $\mathrm{OAB}$.

\section{Micturitions}

\section{Mirabegron Group}

Mean micturitions in a day decreased from an average of $12.02+/-3.10$ per day at baseline to $8.98+/-1.53$ at week 4 and to $8.34+/-1.32$ at week 8 and to $8.02+/-1.13$ per day at week 12 in the mirabegron group. This shows a decreasing trend of mean micturitions in a day after initiation of therapy. The decrease in the mean micturitions in a day from baseline to week 12 was significant as evident by the $P$ value of $<0.001$.

\section{Solifenacin Group}

Mean micturitions in a day decreased from an average of $12.02+/-3.10$ per day at baseline to $8.98+/-1.53$ at week 4 and $8.34+/-1.32$ at week 8 and to $8.02+/-1.13$ per day at week 12 in the solifenacin group. This shows a decreasing trend of incontinence episodes after initiation of therapy. The decrease in the mean micturitions in a day from baseline to week 12 was significant as evident by the $\mathrm{P}$ - value of $<0.001$

\section{Mirabegron VS Solifenacin}

Mean micturitions in a day decreased in both the groups, mirabegron group from 12.02 to 8.02 , solifenacin group from 12.98 to 8.48 at week 12 . But the reduction in numbers between both the groups was not significantly different at week $4(\mathrm{P}<0.1641)$, week $8(\mathrm{P}<0.3747)$ and week $12(\mathrm{P}<$ 0.1401). So based on our present study, both mirabegron and solifenacin are equally effective in reducing the mean micturitions in a day in a patient with $\mathrm{OAB}$.

\section{Micturition Volume \\ Mirabegron Group}

Average volume of each micturition increased from an average of $145.44+/-36.07 \mathrm{~mL}$ per micturition at baseline to $160.22+/-40.93 \mathrm{~mL}$ at week 4 and to $166.94+/-41.95$ $\mathrm{mL}$ at week 8 and to $173.12+/-40.41 \mathrm{~mL}$ per day at week 12 in the mirabegron group. This shows an increasing trend of the average volume of each micturition after initiation of therapy. The increase in volume from baseline to week 12 was significant as evident by the $\mathrm{P}-$ value of $<0.001$. This shows that mirabegron is effective in increasing the average volume of each micturition, thereby increasing the bladder capacity in a patient with OAB. This is in accordance with the Capricon study conducted by Sender Herschorn, Jack Barkin et al. who studied the role of mirabegron for control of $\mathrm{OAB}$ symptoms in 426 patients by comparing mirabegron $25 \mathrm{mg}$ and mirabegron $50 \mathrm{mg}$. There was a significant increase in mean micturition volume in mirabegron $50 \mathrm{mg}$ group (by $20.7+/-2.2 \mathrm{~mL}$ from $159.3+/-2.5 \mathrm{~mL}), \mathrm{P}<0.001$.

\section{Solifenacin Group}

Mean micturition volume increased from an average of $142.52+/-40 \mathrm{~mL}$ per micturition at baseline to $153.44+/$ $39.25 \mathrm{~mL}$ at week 4 and $159.44+/-40.20 \mathrm{~mL}$ at week 8 and to $164.76+/-40.97 \mathrm{~mL}$ per day at week 12 in the solifenacin group. This shows an increasing trend of average volume of each micturition after initiation of therapy. The increase in volume from baseline to week 12 was significant as evident by the $\mathrm{P}$ - value of $<0.001$.

\section{Mirabegron VS Solifenacin}

Mirabegron was more effective in increasing the mean micturition in patients with $\mathrm{OAB}$. There were side-effects 
seen in 16 patients in the mirabegron group, constituting 32 $\%$, in 19 patients in the solifenacin, constituting $38 \%$. The numbers were not large enough to derive any significance. But in our study, the most common side effect in the mirabegron group was hypertension seen in 5 patients. This is in accordance with the Scorpio study conducted by Vik Khul lar, Gerard Amarenco et al. who conducted to determine the efficacy and tolerability of mirabegron in a phase - 3 randomized control trial. Hypertension was the most common side-effect in this study seen in 29 of 429 patients in the mirabegron $50 \mathrm{mg}$ group, constituting $5.9 \%$. The most common side effect in the solifenacin group was dry mouth seen in 7 patients. This is in accordance with the study by Basra R, Kelleher C. which showed the most common sideeffects with solifenacin were dry mouth and constipation. It is observed that the most common side-effect in the mirabegron group was hypertension and the most common side-effect in the solifenacin group was dry mouth.

\section{CONCLUSIONS}

Both mirabegron and solifenacin are effective in controlling the frequency of micturition, decreasing urgency and incontinence episodes and increasing the mean volume of micturition.

Mirabegron is more effective than solifenacin in controlling urgency and incontinence episodes and increasing the mean volume of micturition. There is no statistical difference between the two drugs with regards to a controlling frequency in OAB. Both drugs are well tolerated. Side-effects are seen slightly more with solifenacin. Dry mouth is more common with solifenacin treatment and hypertension with mirabegron.

Data sharing statement provided by the authors is available with the full text of this article at jemds.com.

Financial or other competing interests: None.

Disclosure forms provided by the authors are available with the full text of this article at jemds.com.

\section{REFERENCES}

[1] Abrams P, Cardozo L, Fall M, et al. The standardization of terminology of lower urinary tract function: report from the standardisation sub-committee of the International Continence Society. Neurourol Urodyn 2002;21(2):16778.

[2] Tang DH, Colayco DC, Khalaf KM, et al. Impact of urinary incontinence on healthcare resource utilization, healthrelated quality of life and productivity in patients with overactive bladder. BJU Int 2014;113(3):484-91.

[3] Coyne KS, Sexton CC, Irwin DE, et al. The impact of overactive bladder, incontinence and other lower urinary tract symptoms on quality of life, work productivity, sexuality and emotional well - being in men and women: results from the EPIC study. BJU Int 2008;101(11):1388-95

[4] Coyne KS, Sexton CC, Kopp ZS, et al. The impact of overactive bladder on mental health, work productivity and health-relatedquality of life in the UK and Sweden: results from EpiLUTS. BJU Int 2011;108(9):1459-71.

[5] Coyne KS, Wein A, Nicholson S, et al. Economic burden of urgency urinary incontinence in the United States: a systematic review. J Manag Care Pharm 2014;20(2):13040.

[6] Abrams P, Klevmark B. Frequency volume charts: an indispensable part of lower urinary tract assessment. Scand J Urol Nephrol Suppl 1996;179:47-53.

[7] Gormley EA, Lightner DJ, et al. Diagnosis and treatment of overactive bladder (non-neurogenic) in adults: AUA/SUFU guideline amendment. J Urol 2015;193(5):1572-80.

[8] Ohtake A, Sato S, Sasamata M, et al. The forefront for novel therapeutic agents based on the pathophysiology of lower urinary tract dysfunction: ameliorative effect of solifenacin succinate (Vesicare), a bladder - select ive ant imuscarinic agent, on overactive bladder symptoms, especially urgency episodes. J Pharmacol Sci 2010;112(2):135-41.

[9] EAU guidelines on management of non- neurogenic male LUTS 2021.

[10] Kuo HC, Tzu Chi BT. Comparative of Solifenacin and Mirabegron in Treatment of Overactive Bladder Symptoms in Men After TURP. Clinical trials. gov; U.S National Library of Medicine 2018:NCT03632772. 